\title{
Dexmedetomidine Induces Microglial Activation and Modulates Microglial M1/M2 Polarization in Attenuation of Chronic Morphine Tolerance in Cancer Pain
}

\author{
Jianlong Bu \\ Harbin Medical University Cancer Hospital \\ Wu Xiaohong \\ Harbin Medical University Cancer Hospital \\ Deng Lin \\ Harbin Medical University Cancer Hospital \\ Ma Chao
}

Fourth Affiliated Hospital of Harbin Medical University

Shi Xiaoding

Harbin Medical University Cancer Hospital

PINYI ZHANG ( 0 0511pinyi@163.com )

Harbin Medical University Cancer Hospital https://orcid.org/0000-0002-1807-1507

Wang Guonian

Fourth Affiliated Hospital of Harbin Medical University

\section{Research Article}

Keywords: cancer pain, morphine tolerance, dexmedetomidine, microglia, polarization

Posted Date: January 3rd, 2022

DOI: https://doi.org/10.21203/rs.3.rs-1183447/v1

License: (c) (i) This work is licensed under a Creative Commons Attribution 4.0 International License. Read Full License 


\section{Abstract}

The pro-inflammatory (M1) and anti-inflammatory (M2) status of microglial determines the outcome of neuroinflammation, which contributes to the pathogenesis of chronic morphine tolerance. Studies report that a2-adrenoceptor agonist dexmedetomidine exerts anti-inflammatory effects in inhibiting morphine tolerance in normal and neuropathic pain animals, which has not been studied in cancer pain. Therefore, we investigate the effect of intrathecal DEX on morphine tolerance in cancer pain, and whether dexmedetomidine functions via modulating microglial activation and M1/M2 polarization. 54 Wistar rats with intrathecal catheterization were treated by morphine for 10 days. Test groups received intrathecal a2adrenoceptor agonist dexmedetomidine or antagonist MK-467. The mRNA levels of TLR4 and NF-KB were tested by RT-PCR. The protein levels of TLR4, NF-KB, Iba-1, iNOS, CD206 were quantifed using Western blotting, and IL-10 and TNF-a were examined by ELISA. Dexmedetomidine attenuates mechanical threshold and thermal latency, and increased the expression of TLR4 and NF-KB in morphine tolerance of cancer pain. Dexmedetomidine attenuates mechanical and thermal nociception in morphine tolerance in cancer pain rats. Intrathecal DEX pre-treatment significantly increased the protein levels of microglia maker Iba-1, M2 marker CD206 and anti-inflammatory factor IL-10, while had no evident influence on the pro-inflammatory factor TNF-a and M1 marker iNOS in morphine tolerance. Our findings suggest that intrathecal dexmedetomidine attenuates morphine tolerance in cancer pain via a2-adrenoceptor pathway. Furthermore, dexmedetomidine upregulates TLR4/NF-KB pathway and induces microglia activation in chronic morphine tolerance of cancer pain. The anti-inflammatory effect of dexmedetomidine might be exerted by inducing microglia M2 polarization and increasing anti-inflammatory factor IL-10.

\section{Highlights}

1. Intrathecal DEX increases the expression of TLR4/NF-KB pathway and induces microglia activation in chronic morphine tolerance of cancer pain.

2. DEX exerts the anti-inflammatory effect to attenuate the development of chronic morphine tolerance in cancer pain by inducing microglia M2 polarization and increasing production of anti-inflammatory factor IL-10.

\section{Introduction}

Cancer pain is one of the most serious conditions that severely impairs patients' quality of life [1]. Morphine is a prototypical opiate analgesic commonly used in the treatment for cancer pain. However, with long-term use, the development of analgesic tolerance becomes a major problem [2]. In addition to neuronal mechanisms, microglial activiation has emerged as a potentially significant new mechanism of morphine tolerance. Recent studies demonstrate that microglia-mediated neuroinflammation plays a double-edged role in the pathological processes of chronic morphine treatment [3]. There are basically two polarized states of activated microglia, the classical deleterious 'M1' phenotype and the alternative neuroprotective 'M2' phenotype [4]. Imbalanced microglial polarization, in the form of excessive activation of M1 microglia and dysfunction of M2 microglia, promotes the development of 
neuroinflammatory response in sustained morphine exposure. Thus, regulating the microglia polarization provides us a new target in the treatment for morphine tolerance.

A number of signaling molecules, including Toll-like receptor 4 (TLR4) and Nuclear Factor-kappa B (NF$\mathrm{KB})$, are critical to the modulation of microglial activation and neuroinflammation. TLR4 expresses in a wide variety of cells and recognizes the invariant molecular structures of pathogens and participates in the innate immunity [5]. The central immune signaling initiated by TLR4, resulting in the activation of downstream mediators, including the transcription factor nuclear factor (NF)-kB, which increases the production of pro-inflammatory cytokines, such as tumor necrosis factor (TNF)- $a$, interleukin (IL)-1 $\beta$, and anti-inflammatory cytokines such as IL-10 [6]. Accumulating evidence indicates that TLR4-mediated NF$\mathrm{KB}$ activation in the spinal cord is implicated in opioid tolerance, hyperalgesia, and physical dependence [7][8].

Microglial cells express mRNA encoding a2-adrenoceptor (a2-AR) linked to the signal transduction cascade TLR4/NF-KB pathway. In microglia, TLR4 pathway mediates the activation of iNOS induced by LPS. It has been found that the inflammatory response of microglia could be weakened by inhibiting TLR4 pathway [9]. Dexmedetomidine (DEX) is a highly selective agonist of a2-adrenoceptor (a2-AR) with sedative properties and analgesic potency. Numerous studies have shown that DEX exerts an antiinflammatory effect in many conditions of neural disorders [10][11]. Furthermore, in vitro, a recent study demonstrates that DEX alleviates neuroinflammation through influencing microglial M1/M2 polarization [12]. It has been reported that administration of a2-AR agonists attenuated the progress of morphine analgesic tolerance in normal rats and neuropathic pain rats, possibly by preventing the morphineinduced neuroinflammatory activities [13][14]. Moreover, the role of a2-AR DEX in neuroinflammatory response of non-cancer pain have been investigated involving down-regulating TLR4/NF-KB signaling pathway [15][16]. However, the mechanism underlying the effect of DEX on chronic morphine tolerance in cancer pain has not been studied.

In this study, we aim to investigate the effect of intrathecal DEX injection on the development of morphine tolerance in cancer pain treatment, and whether this DEX-induced effect is exerted by modulating microglia polarized activities and propose that TLR4/NF-KB signaling pathway might be involved in this process.

\section{Materials And Methods}

\section{Animals}

Adult male Wistar rats (6-7 weeks, $180-200 \mathrm{~g}, \mathrm{n}=60$ ) obtained from Harbin Medical University Laboratories (Harbin, China) were housed at a constant ambient temperature of $22 \pm 1^{\circ} \mathrm{C}$ under a $12-\mathrm{h}$ light-dark cycle with ad libitum access to water and food. All animal experiments complied with the policies and recommendations of the International Association for the Study of Pain, and the National Institutes of Health $(\mathrm{NIH})$ guidelines for the handling and use of laboratory animals. The experimental 
protocol was approved by the Animal Care and Use Committee of Harbin Medical University (Harbin, China).

\section{Intrathecal catheterization}

Intrathecal (i.t.) catheter implantation was performed following the procedures described by previous studies with modifications [17]. After inhalation anesthetized with sevoflurane (2-3\% in oxygen, Abbott Pharmaceutical Co. Ltd., Lake Bluff, IL, USA), the animal was placed in a prone position on the experimental table, and the bilateral spurs were found. A midline skin incision was made in the lumbar region (L3-L5), and the intervertebral membrane between L3 and L4 was exposed. A $25 \mathrm{G}$ needle was then used to puncture the membrane, and the catheter (Polyethylene PE 10, Becton Dickinson, Franklin Lake, NJ) was inserted into the subarachnoid space about $2.0 \mathrm{~cm}$. The catheter was fixed to the muscle, and the other end was tunneled rostrally underneath the skin to exit $1.5 \mathrm{~cm}$ in the occipital region. The incisions were then closed in layers using 4-0 silk.

The rats were allowed to recover for 5 days in separate cage before behavioral testing. Rats showing hind limbs paralysis within $30 \mathrm{~s}$ after i.t. administration of $2 \%$ lidocaine $(10 \mu \mathrm{l})$ suggested successful i.t. catheterization. The position of the catheter was checked postmortem. Rats displaying any abnormal neurological signs were excluded from experiments.

\section{Experimental design}

According to our previous studies [18], after intrathecal catheterization, each rat was implanted with Walker 256 breast carcinoma cells (provided by the Cancer research institute of Heilongjiang, Harbin, China) on the plantar region of right hindpaw. The Walker 256 cell culture procedure was conducted as described previously [19]. On day 5 post-inoculation, a significant proliferation of tumor cells was detected with a development of hyperalgesia and allodynia of the hind paw, as indicated by comparing the TWL and MWT to the values before inoculation in all rats. This indicates a successful establishment of cancer pain model.

A total of 54 tumor-bearing rats were randomly allocated to 6 groups ( $n=9$ for each group). All drugs were administered twice daily (9:00 am and 5:00 pm) for 10 days (day 1 to day 10). To induce morphine analgesic tolerance, a 10-day cumulative dosing regimen was used. The rats in morphine group were injected with intrathecal (i.t.) normal saline $10 \mu \mathrm{l}$ and subcutaneous (s.c.) morphine sulfate (Northeast Pharmaceutical Group Co., Ltd., Harbin, China) $10 \mathrm{mg} / \mathrm{kg} / \mathrm{mL}$ (vehicle+morphine group). The analgesic effects of morphine were measured by the tail-flick test. Drugs were delivered as follows: in separate group, rats received i.t. injection of dexmedetomidine $(10 \mu \mathrm{g} / \mathrm{kg}, 20 \mu \mathrm{l}$, Heng Rui Pharmaceutical Co., Ltd. Jiangsu, China) and s.c. injection of morphine (DEX+morphine group), or i.t. injection of $a_{2}-A R$ antagonist MK-467 $(0.25 \mathrm{mg} / \mathrm{kg}, 10 \mu \mathrm{l}$, USA) $30 \mathrm{~min}$ prior to DEX and s.c. injection of morphine (MK$467+D E X+$ morphine group). DEX was injected $30 \mathrm{~min}$ prior to morphine. The rats received i.t. $10 \mu \mathrm{l}$ and s.c. $1 \mathrm{ml} / \mathrm{kg}$ injection of normal saline served as control group (vehicle+saline group). Furthermore, to test the effects of DEX on cancer pain, the rats received i.t. injections of DEX (DEX+saline group) or MK-467 30 min prior to DEX (MK-467+DEX+saline group). 


\section{Behavioral Tests}

Rats were acclimated within plexiglass enclosures on a clear glass plate maintained at $26 \pm 0.5^{\circ} \mathrm{C}$. Behavioral assays were performed before drug administration (at baseline) on day 0 and 30 min after morning drug delivery from day 1 to day 10. Mechanical withdrawal threshold (MWT) was measured by applying von Frey filaments (Stoelting Company, Wood Dale, IL, USA) and used a staircase method. The MWT was defined as the lowest filament in grams when the filament aroused a positive reaction (licking or lifting of hindpaw), and a $60 \mathrm{~g}$ maximum was imposed as a cutoff to avoid tissue damage. Three measurements were performed on each rat randomly beginning with the left or right paw, and the mean value of MWT from both hind paws was used. Thermal withdrawal latency (TWL) was determined for each rat using a hot plate apparatus in a plastic cylinder (Technology \& Market CORP, Chengdu, China). Rats were individually placed on the hotplate $\left(52^{\circ} \mathrm{C}\right)$, and latency was defined is the interval between exposure to the hotplate and positive reaction (licking or lifting of the unilateral hindpaw) due to heat stimulation. The maximal cut-off time was $30 \mathrm{~s}$ in order to avoid damage to the paw. The average latency was calculated from 3 measurements taken at 5 min intervals.

\section{Reverse Transcription Quantitative Polymerase Chain Reaction (RT-qPCR)}

The animals were sacrificed at a designated time point. RT-PCR was performed using lumbar L3-L5 segments of DRG ipsilateral to the tumor cell injections. Total RNA was isolated using TRIzol (Invitrogen, Carlsbad, CA), and a $2 \mu \mathrm{g}$ aliquot was used for complementary deoxyribonucleic acid (cDNA) synthesis using the Transcriptor First Strand cDNA Synthesis Kit (Roche, Basel, Switzerland). The cDNA was used as a template for RT-PCR amplification using Fast Start Universal SYBR Green Master Mix (Roche, Basel, Switzerland). Primers for TLR4, NF-KB and GAPDH (glyceraldehyde-3-phosphate dehydrogenase) were designed by Primer Express software (Applied Biosystems, Inc., Carlsbad, CA) and were TLR4 (forward: 5'CAGGGACTTTATTCCTGGTGTAG-3', reverse: 5'-CACCAACGGCTCTGGATAAA-3'); NF-kB (forward: 5'GGTTACGGGAGATGTGAAGATG-3', reverse: 5'-GTGGATGATGGCTAAGTGTAGG-3'); GAPDH (forward: 5'GGTGGACCTCATGGCCTACAT-3', reverse: 5'-CTCTCTTGCTCTCAGTATCCTTGCT-3'). Quantitative PCR amplification was performed according to the manufacturer's instructions ( 30 seconds at $95^{\circ} \mathrm{C}, 40$ cycles of 5 seconds at $95^{\circ} \mathrm{C}$ and 30 seconds at $55^{\circ} \mathrm{C}$ ) using an $\mathrm{ABI} 7500$ fast real-time PCR system (Applied Biosystems, Inc., Carlsbad, CA).

\section{Western blotting}

Western blotting was performed as our previous procedures [18]. In brief, samples (L3-L5 segments of DRG) were collected and washed with ice-cold phosphate buffered saline (PBS) before lysed in radio immunoprecipitation assay (RIPA) lysis buffer (Solarbio, Beijing, China). Then, whole sample lysates were collected for homogenization and centrifugeration, and the protein concentration of the supernatant was measured by the Bradford method. The proteins were electrophoretically transferred onto polyvinylidene difluoride (PVDF) membranes, which were blocked with $3 \%$ non-fat dry milk for $1 \mathrm{~h}$ and incubated overnight at $4^{\circ} \mathrm{C}$ with primary antibodies for TLR4 (ABclonal, 1:1 000), NF-KB (Immunoway, 1:2 000) Iba- 
1(Abcam, 1:500), iNOS (ABclonal, 1:1 000), CD206 (ABclonal, 1:1 000) and GAPDH (ABclonal, 1:500, performed as a loading control). Then the filters were developed using ECL reagents (Meilunbio, Dalian, China) with secondary antibodies [goat anti-mouse or goat anti-rabbit horseradish peroxidaseconjugated (HRP), 1:3 000, Millipore Chemicom, USA]. Densitometric scanning was used for quantitative analysis.

\section{Enzyme-linked immunosorbent assay}

After the rats were sacrificed under deep anesthesia with pentobarbital sodium (1\% in NS, $50 \mathrm{mg} / \mathrm{kg}$, i.p.), the lumbar spinal cord segments were collected. The supernatants of tissue homogenates were collected and analyzed using enzyme-linked immunosorbent assay (ELISA) kits (Senbeijia, Nanjing, China) for IL10 and TNF-a according to the manufacturer's instructions.

\section{Immunohistochemistry}

The rats were deeply anesthetized and perfused through the ascending aorta with normal saline, followed by $4 \%$ paraformaldehyde in $0.1 \mathrm{~mol} / \mathrm{L}$ phosphate buffer. Subsequently, the lumbar (L3-5) SC was removed and post-fixed in the same fixative at $4^{\circ} \mathrm{C}$ for $24 \mathrm{~h}$, and then the fixative was replaced by $30 \%$ sucrose in PBS over two nights. Transverse spinal sections $(20 \mu \mathrm{m})$ were cut on a cryostat and prepared for immunofluorescence staining. Sections, after a randomly selection and PBS wash, were blocked with $5 \%$ goat serum in $0.3 \%$ Triton $\mathrm{X}-100$ for $1 \mathrm{~h}$ at $37^{\circ} \mathrm{C}$, and incubated with primary antibodies for TLR4 (ABclonal, 1:100) and NF-KB (Immunoway, 1:100) overnight at $4^{\circ} \mathrm{C}$. After washing with PBS for 3 times, the sections were incubated in goat anti-rabbit IgG (1:100) for NF-KB p65 or goat anti-mouse lgG (1:100) for TLR4 for $1 \mathrm{~h}$ at $37^{\circ} \mathrm{C}$. The morphological details were examined under an inverted microscope (Olympus Corporation, Tokyo, Japan). The positive area of the images was digitized and subjected to color threshold analysis using NIH ImageJ software version 2.1 (National Institutes of Health, Bethesda, MD, USA).

\section{Statistical analyses}

Data were analyzed by the Statistical Product for Social Sciences (SPSS version 23.0). Data were presented as the mean $\pm S D$. For behavioral data, the difference between groups was determined by Twoway Repeated Measures Anova followed by Student $t$ test with Bonferroni correction. TLR4, NF-KB, Iba-1, iNOS, CD206, TNF-a and IL-10 expression were analyzed using one-way ANOVA followed by Student $t$ test with Bonferroni correction for multiple comparisons. For the data from behavioral tests, RT-PCR, western blotting and ELISA, we performed 7 comparisons: vehicle+saline versus the other 5 groups,

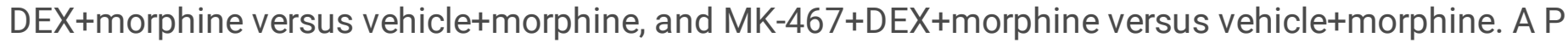
value less than the Bonferroni-corrected threshold of 0.0071 (0.05/7) was defined as statistically significant. For the data from immunohistochemistry, we performed 5 comparisons: vehicle+saline versus $D E X+m o r p h i n e$, vehicle+morphine, and MK-467+DEX+morphine group. A P value less than the Bonferronicorrected threshold of $0.01(0.05 / 5)$ was defined as statistically significant.

\section{Results}




\section{Establishment of Morphine Tolerance in Cancer Pain}

Consistent with the results of our previous research [18], 5 days after injection of Walker 256 tumor cells, a significant development of mechanical and thermal sensitivity indicated painful behaviors caused by cancer was observed in rats. Based on the 7-day morphine treatment in our previous research [18], chronic morphine analgesic tolerance in cancer pain was established using a 10-day morphine administration. As shown in Figure 1-1 and Figure 1-2, from day 4 to day 10, the MWT and TWL in vehicle+morphine group had no significant change compared with control group, indicating that chronic morphine administration elicited notable analgesic tolerance in the rats with cancer pain.

Dexmedetomidine Attenuates Mechanical Hyperalgesia and Thermal Nociception in Development of Morphine Tolerance in Cancer Pain Rats

Regarding the effect of dexmedetomidine on mechanical hyperalgesia and thermal nociception in the development of morphine tolerance in cancer pain, we also obtained similar results as our previous research [18]. As shown in Figure 1-1 and Figure 1-2, compared with vehicle+morphine group, MWT $(P<0.007)$ and TWL $(P<0.007)$ in DEX+morphine group significantly increased from day 3 to day 10 , suggesting that pre-administration of DEX could strengthened the analgesic effect of morphine. Although MWT (days 3-10: all $P<0.007$ ) and TWL (days 3-10: all $P<0.005$ ) values of DEX-pretreated rats (DEX+morphine group) reduced substantially from day 4 to day 10 compared with day 1 , the analgesic effect remained significantly higher than control group (days 1-10: all $P<0.007$ ) on each day. Thus, preadministration of DEX could attenuate the formation of morphine analgesic tolerance $(P<0.007)$, which could be reversed by a2-AR antagonist MK-467.

\section{Dexmedetomidine Promotes Microglial Activation in DRG of Morphine Tolerant Cancer Pain Rats}

Western blotting was performed to determine the protein level of Ibal-1 in the L3-L5 spinal cord. As presented in Figure 2, compared with the vehicle+morphine group, pre-administion of DEX (DEX+morpnine group) significantly increased Ibal-1 ( $1.8983 \pm 0.14289$ vs. $1.0553 \pm 0.15355, P<0.001)$, suggesting that DEX promoted microglial activation in morphine tolerance of cancer pain. No significant difference was found between MK-467+DEX+morphine group and vehicle+morphine group, suggesting that the a2-AR antagonist MK-467, 30 min prior to DEX administration could block the effect of DEX on upregulation of Ibal-1 expression.

\section{Dexmedetomidine Up-regulates TLR4/NF-kB Signaling Pathway in DRG of Morphine Tolerant Cancer Pain Rats}

RT-PCR was used to determine the mRNA expression of TLR4 and NF-KB in the L3-L5 DRG of cancer pain rats. As presented in Figure 3-1 and Figure 3-2, pre-administion of DEX (DEX+morpnine group) significantly increased TLR4 $(0.73 \pm 0.19$ vs. $1.15 \pm 0.09, P=0.001<0.007)$ and NF-KB $(0.77 \pm 0.28$ vs. $1.65 \pm 0.37, \mathrm{P}=0.001<0.007)$ mRNA expression in $\mathrm{DRG}$ compared with morphine administration alone. No 
significant difference was found between MK-467+DEX+morphine group and vehicle+morphine group, suggesting that the a2-AR antagonist MK-467, 30 min prior to DEX administration could block the effect of DEX on morphine-induced TLR4 and NF-KB mRNA expression. Compared with the control group, DEX administration (DEX+saline group) significantly decreased the mRNA expression of TLR4 ( $※ P=0.001)$ and $\mathrm{NF}-\mathrm{KB}(※ \mathrm{P}=0.002)$ in cancer pain rats. Compared with the control group, MK-467+DEX+saline group $(※ \mathrm{P}<0.007)$ or vehicle+morphine group $(※ \mathrm{P}=0.003)$ significantly decreased NF-KB mRNA expression in cancer pain rats.

Furthermore, western blotting was performed to determine the protein level of TLR4 and NF-KB in the L3L5 DRG. As shown in Figure 4-1 and Figure 4-2, DEX-pretreated (DEX+morpnine group) rats displayed a significant increase in TLR4 ( $0.49 \pm 0.10$ vs. $1.04 \pm 0.26, P=0.001<0.007)$ and NF-KB $(1.25 \pm 0.21$ vs. $2.07 \pm 0.44, P=0.002<0.007)$ expression in $D R G$ compared with rats in vehicle+morphine group. The effect of DEX on morphine-induced TLR4 and NF-KB protein expression was abolished by the a2-AR antagonist MK-467, and there was no difference between MK-467+DEX+morphine group and vehicle+morphine group.

Immunohistochemistry revealed TLR4 (Figure 5-1) and NF-KB (Figure 5-2) protein expression in DRG. Compared with morphine group, pretreatment with $D E X$ ( $D E X+$ morphine group) significantly increased TLR4 ( $0.27 \pm 0.04$ vs. $0.57 \pm 0.13, P=0.006<0.01)$ and NF-KB $(0.24 \pm 0.04$ vs. $0.40 \pm 0.06, P=0.002<0.01)$ protein expression in DRG. MK-467 abrogated the effect of DEX on morphine-induced TLR4 and NF-KB expression, and there was no difference between MK-467+DEX+morphine group and vehicle+morphine group.

Dexmedetomidine Modulates Microglial Activity towards the M2 Phenotype in the Spinal Cord of Morphine Tolerant Cancer Pain Rats

To evaluate the effects of DEX on inflammatory factors and M1/M2 phenotypes in morphine tolerant cancer pain rats, western blot and ELISA analysis were performed. Compared with morphine group, pretreatment with intrathecal DEX significantly increased the protein levels of CD206 $(0.57 \pm 0.06 \mathrm{vs}$. $0.82 \pm 0.01, P=0.002<0.007)$ (Figure 6-1) and IL-10 ( $P=0.003<0.007)$ (Figure 7-1) in morphine tolerant cancer pain rats, while had no evident influence on the protein expression of iNOS (Figure 6-2) and TNF-a (Figure 7-2). This effect of DEX could be blocked by the a2-AR antagonist MK-467.

\section{Discussion}

In this study, we assessed the effect of DEX on chronic morphine tolerance in a rat model of cancer pain. Intrathecal injection of DEX enhanced morphine analgesia and attenuated the development of chronic morphine tolerance. DEX promoted microglial activation and upregulated the expression of TLR4/NF-KB signaling pathway. Moreover, DEX exhibited anti-inflammatory effect in attenuation of chronic morphine tolerance by modulating microglial activity towards the M2 phenotype, characterized by higher expression of anti-inflammatory factor IL-10 and M2 maker CD206. All these effect of DEX could be blocked by a selective a2-AR antagonist MK-467. 
Morphine-induced microglia-mediated neuroinflammation is a complex molecular system involving various signaling pathways. Accumulating evidence demonstrates that TLR4/NF-KB pathway plays a critical role in morphine-induced microglia activation [7], and inhibition of TLR4/NF-KB has been proven to be effective on attenuating morphine tolerance [16]. However, to date, almost all these researches on TLR4/NF-KB pathway in morphine tolerance have been performed based on normal animals or noncancer pain models. Few studies have investigated the role of TLR4/NF-KB signaling in morphine tolerance on cancer pain model. Surprisingly, inconsistent with most of previous studies based on noncancer pain models, our study revealed that intrathecal injection of DEX promoted microglial activation and increased the expression of TLR4 and NF-KB in DRG of morphine tolerant rats with cancer pain, and this effect could be reversed by $a_{2}-A R$ antagonist MK-467. These findings showed that DEX-induced attenuation in morphine tolerance of cancer pain did not exert via suppressing TLR4/NF-KB pathway.

Numerous studies indicate that TLR4 promotes inflammatory responses that are correlated with the pathology of pain [20][21]. It has been reported that DEX significantly decreased upregulation of TLR4 expression in spinal cord of rats with monoarthritis [22]. Similarly, in our study, DEX administration (DEX+saline group) decreased the mRNA and protein expression of TLR4 in DRG compared with nontreated rats (vehicle+saline group), suggesting that DEX could suppress the inflammatory responses via down-regulating TLR4 expression in cancer pain. However, in the state of morphine tolerance, our results showed that intrathecal administration of DEX significantly increased TLR4 and NF-KB expression in DRG of the rats with cancer pain on both mRNA and protein level. This finding suggested that DEX increased the morphine-induced microglial activation characterized in upregulating TLR4/NF-KB pathway. Previous studies have shown TLR4 antagonist LPS-RS alleviates chronic neuropathic pain caused by nerve chronic constriction injury at the first administration, while fails at a subsequent administration [23]. In the post-inflammatory stage, LPS-RS also fails to inhibit the TLR4 activation that occurred after the stimulus ceased [24]. These results indicate that TLR4 may not play a major effect during the whole chronic neuroinflammatory response. On the other hand, a previous study has reported that in a morphine tolerance model, microglial activation is caused by a TLR4-independent mechanism [25]. Moreover, Mattioli and colleagues [26] demonstrated that TLR4 was not required for the development of morphineinduced analgesic tolerance, hyperalgesia, or physical dependence. Herein, we speculate that the inhibiting effect of DEX on morphine tolerance in cancer pain may not regulated via TLR4/NF-KB pathway, whereas involve other mechanism of central neuro-inflammatory responses.

Long-term application of morphine has been found to enhance neuroimmune reactivity, involving microglial activation and proinflammatory cytokine production, and then resulted in analgesic tolerance. Activated microglial cells exist in M1 phenotype and M2 phenotype. M1 microglia aggravates tissue damage by producing destructive pro-inflammatory factors such as IL-1 $\beta$, TNF-a, while M2 microglia promotes injury repair by releasing anti-inflammatory factors such as IL-4, IL-10 and some neurotrophic factors [27]. Furthermore, the ratio between microglial M1 and M2 phenotypes affects the development trend of neuroinflammatory response, which could be intensified by increase of the M1/M2 ratio [28]. Recently, a study assessed the effects of DEX on microglia polarization [12], revealing that treatment with 
DEX increased the expression of anti-inflammatory factor IL-10 and M2 phenotypic marker CD206, while decreased the levels of pro-inflammatory factors TNF-a and M1 phenotypic marker iNOS. IL-10 plays a pivotal role in morphine tolerance and regulates the production of proinflammatory cytokines, including IL-1 $\beta, I L-6, T N F-\alpha$ [29][30]. Consistently, in this study of cancer pain, we found that intrathecal DEX increased the expression of anti-inflammatory factor IL-10 and M2 phenotypic marker CD206, while had no significant influence in the levels of pro-inflammatory factors TNF- $\alpha$ and M1 phenotypic marker iNOS, indicating that DEX-modulated microglial activation may due to microglia polarisation to the M2 phenotype and promotion of the anti-inflammatory cytokines IL-10, consequently inhibiting morphineinduced chronic neuro-inflammatory responses of analgesic tolerance. M1/2 microglia polarization has not been studied previously in the context of chronic morphine administration. In cancer pain management, prolong morphine exposure stimulated the microglial activities and induces analgesic tolerance. In our investigation, the increased expression of M2-microglia in the spinal cord could be interpreted as an effort to resolve the neuroinflammation caused by chronic morphine administration.

The mechanisms of microglia polarization involve various molecules and signaling pathways. Parthenolide, known to affect intracellular microglial inflammation pathways, like p-38 and ERK $1 / 2$ has promoted spinal M2 microglia/macrophage polarization and relieved pain in a rat model of neuropathy [31]. A recent study demonstrated that dexmedetomidine induces microglial polarization towards M2 phenotype by inhibiting phosphorylation of ERK1/2 in vivo [32]. Dexmedetomidine decreased the expression of TNF- $a$ and inhibited activation of ERK1/2 in the lung tissues of cecal ligation and puncture (CLP)induced septic rats [33]. Dexmedetomidine also inhibited the expression of purinergic receptor 7 (P2X7R) and ERK phosphorylation that attenuated chronic neuropathic pain [34]. Considering the role of activated microglia in both neuropathic pain and opioid-induced neuroinflammation, drugs that promote microglia M2-conversion could be beneficial in attenuating morphine tolerance. In our study, further researches need to be carried out to explore the molecular mechanisms and signaling pathways underlying DEX-induced M2 polarization and anti-inflammatory cytokine releasing.

There are some limitations to this study. Firstly, we only measured the expression of TLR4/NF-KB signaling pathway and the activation of central immune signals at a single time point (day 10). It could not fully reflect the influence of DEX on the central immune system during the whole process of morphine tolerance development in cancer pain. Secondly, our research for the first time showed that in the development of morphine treatment in cancer pain, DEX inhibited morphine tolerance by promoting microglial activation and M2 polarization, and meanwhile DEX exerted the anti-inflammatory effects by releasing anti-inflammatory factor IL-10. However, we only detected the expression levels of M1 and M2 marker proteins in microglia from a molecular perspective. A cytological method would be carried out to better detect the changes in the ratio of microglia M1 and M2 phenotypes. Third, we did not investigate the central immune signaling pathways and molecular mechanisms involved in the DEX-induced attenuation of morphine tolerance in cancer pain. Further studies would be required to address these limitations. 
In conclusion, the present study demonstrated that intrathecal injection of DEX augmented the analgesic effect of morphine and attenuated the development of morphine tolerance in cancer pain via the a2-AR pathway. The anti-inflammatory effect of DEX in morphine tolerance of cancer pain involved in modulating microglia M2 phenotype and promoting releases of IL-10. Moreover, co-administration of DEX and morphine induced microglial activation and increased the expression of TLR4/NF-KB pathway in morphine tolerant rats with cancer pain. It provides an new insight into the fact that the inhibitory effect of DEX on morphine tolerance in cancer pain may be regulated not through suppressing TLR4/NF-KB pathway but by modulating microglia M2 polarization.

\section{Declarations}

\section{Ethics approval and consent to participate}

The experimental protocol was approved by the Animal Care and Use Committee of Harbin Medical University (Harbin, China).

\section{Consent for publication}

Not applicable

\section{Availability of data and materials}

Data sharing not applicable to this article as no datasets were generated or analysed during the current study.

\section{Funding}

This work was supported by a grant from the National Natural Science Foundation of China (Grant No. 8177051154) and the Haiyan Research Fund of Harbin Medical University Cancer Hospital (JJQN202113).

\section{Competing interests}

The authors declare that they have no competing interests.

\section{Author Contribution Statement}

Pinyi Zhang. Conceptualization, Data curation, Formal analysis, Methodology, Writing - original draft. Jianlong Bu. Conceptualization, Formal analysis, Methodology, Writing - review \& editing. Xiaohong Wu. Conceptualization, Methodology, Writing - review \& editing. Lin Deng. Data curation, Formal analysis. Chao Ma: Data curation, Formal analysis. Xiaoding Shi. Data curation, Formal analysis. Guonian Wang. Conceptualization, Funding acquisition, Supervision, Writing - review \& editing.

\section{Author Contribution Statement}




\section{References}

1. Van Den Beuken-Van Everdingen MH, De Rijke JM, Kessels AG, Schouten HC, Van KM, Patijn J. Prevalence of pain in patients with cancer: a systematic review of the past 40 years. Ann Oncol. 2007, 18(9):1437-1449.

2. Kalso E, Edwards JE, Moore RA, McQuay HJ. Opioids in chronic non-cancer pain: systematic review of efficacy and safety. Pain 2004,112(3):372-80.

3. V. Jokinen, Y. Sidorova, H. Viisanen, I. Suleymanova, H. Tiilikainen, Z. Li, T.O. Lilius, K. Mätlik, J.E. Anttila, M. Airavaara, L. Tian, P.V. Rauhala, E.A. Kalso, Differential spinal and supraspinal activation of glia in a rat model of morphine tolerance. Neuroscience. 2018.

4. Durafourt, B.A.; Moore, C.S.; Zammit, D.A.; Johnson, T.A.; Zaguia, F.; Guiot, M.C.; Bar-Or, A.; Antel, J.P. Comparison of polarization properties of human adult microglia and blood-derived macrophages. Glia. 2012, 60, 717-727.

5. Hutchinson MR, Northcutt AL, Hiranita T, et al. Opioid activation of toll-like receptor 4 contributes to drug reinforcement. J. Neurosci. 2012, 32(33):11187-11200.

6. Chen YL, Law PY, Loh HH. Nuclear factor kappaB signaling in opioid functions and receptor gene expression. J Neuroimmune Pharmacol. 2006, 1: 270-279.

7. Liying Bai, Caihong Zhai, Kun Han, et al. Toll-like receptor 4-mediated nuclear factor-kB activation in spinal cord contributes to chronic morphine-induced analgesic tolerance and hyperalgesia in rats. Neurosci Bull, 2014, 30(6): 936-948.

8. DeLeo JA, Tanga FY, Tawfik VL. Neuroimmune activation and neuroinflammation in chronic pain and opioid tolerance/hyperalgesia. Neuroscientist. 2004, 10:40-52.

9. Zhou X-Y, Liu J, Xu Z-P, Fu Q, Wang P-Q, Zhang H. Dexmedetomidine inhibits the lipopolysaccharidestimulated inflammatory response in microglia through the pathway involving TLR4 and NF-KB. Kaohsiung J Med Sci. 2019;35:750-756.

10. Hayashi Y, Guo TZ, Maze M. Hypnotic and analgesic effects of the alpha 2-adrenergic agonist dexmedetomidine in morphine-tolerant rats. Anesth Analg, 1996,83:606-10.

11. Yan H, Yi L, Lei Z, Jia Y, Jue J, Hong J. Perineural Dexmedetomidine attenuates inflammation in rat sciatic nerve via the NF-KB pathway. Int J Mol Sci. 2014;15:4049-4059.

12. Qiu Z, Lu P, Wang K, et al. Dexmedetomidine Inhibits Neuroinflammation by Altering Microglial M1/M2 Polarization Through MAPK/ERK Pathway. Neurochem Res. 2020, 45(2):345-353.

13. Gursoy S, Ozdemir E, Bagcivan I, Altun A, Durmus N. Effects of alpha 2-adrenoceptor agonists dexmedetomidine and guanfacine on morphine analgesia and tolerance in rats. Ups J Med Sci. 2011, 116(4):238-46.

14. Nakagawa T, Nagayasu $\mathrm{K}$, Nishitani $\mathrm{N}$, et al. Yokukansan inhibits morphine tolerance and physical dependence in mice: the role of a囚A-adrenoceptor. Neuroscience. 2012, 227:336-349. 
15. Ledeboer A, Gamanos M, Lai W, Martin D, Maier SF, Watkins LR, et al. Involvement of spinal cord nuclear factor kappaB activation in rat models of proinflammatory cytokine-mediated pain facilitation. Eur J Neurosci. 2005, 22:1977-1986.

16. Tegeder I, Niederberger E, Schmidt R, Kunz S, Guhring H, Ritzeler O, et al. Specific Inhibition of kappaB kinase reduces hyperalgesia in inflammatory and neuropathic pain models in rats. J Neurosci. 2004, 24:1637-1645.

17. Storkson RV, Kjorsvik A, Tjolsen A, Hole K. Lumbar catheterization of the spinal subarachnoid space in the rat. J Neurosci Methods. 1996, 65: 167-72.

18. Zhang P, Bu J, Wu X, Deng L, Chi M, Ma C, Shi X, Wang G. Upregulation of $\mu$-Opioid Receptor in the Rat Spinal Cord Contributes to the a2-Adrenoceptor Agonist Dexmedetomidine-Induced Attenuation of Chronic Morphine Tolerance in Cancer Pain. J Pain Res. 2020, 15;13:2617-2627.

19. P. Brigatte, S. Sampaio, V. Gutierrez, Walker 256 tumor-bearing rats as a model to study cancer pain, J. Pain. 2007, 8:412-421.

20. Cao L, Tanga FY, Deleo JA. The contributing role of CD14 in toll-like receptor 4 dependent neuropathic pain. Neuroscience. 2009, 158(2):896-903.

21. Ramachandran R, Wang Z, Saavedra C, DiNardo A, Corr M, Powell SB, Yaksh TL. Role of Toll-like receptor 4 signaling in mast cell-mediated migraine pain pathway. Mol Pain. $2019 \mathrm{Jan}-\mathrm{Dec}$,

22. Ji D, Zhou Y, Li S, Li D, Chen H, Xiong Y, Zhang Y, Xu H. Anti-nociceptive effect of dexmedetomidine in a rat model of monoarthritis via suppression of the TLR4/NF-KB p65 pathway. Exp Ther Med. 2017, 14(5):4910-4918.

23. Hutchinson, M.R., Zhang, Y., Brown, K. Non-stereoselective reversal of neuropathic pain by naloxone and naltrexone: involvement of toll like receptor 4 (TLR4). Eur. J. Neurosci. 2008, 28, 20-29.

24. Christina, A.C., Darren, S.D., Jennifer, A.S. Spinal TLR4 mediates the transition to a persistent mechanical hypersensitivity after the resolution of inflammation in serum-transferred arthritis. Pain. 2011, 152, 2881-2891.

25. Fukagawa, H., Koyama, T., Kakuyama, M., Fukuda, K. Microglial activation involved in morphine tolerance is not mediated by toll-like receptor 4. J. Anesth. 2013, 27, 93-97.

26. Mattioli TA, Leduc-Pessah H, Skelhorne-Gross G, Nicol CJB, Milne B, et al. Toll-Like Receptor 4 Mutant and Null Mice Retain Morphine-Induced Tolerance, Hyperalgesia, and Physical Dependence. PLoS ONE 2014, 9(5): e97361.

27. Hu X, Li P, Guo Y, Wang H, Leak RK, Chen S, Gao Y, Chen J. Microglia/macrophage polarization dynamics reveal novel mechanism of injury expansion after focal cerebral ischemia. Stroke. 2012, 43(11):3063-3070.

28. Sun D, Yu Z, Fang X. LncRNA GAS5 inhibits microglial M2 polarization and exacerbates demyelination. Embo Rep. 2017, 18(10):1801-1816.

29. Tai YH, Tsai RY, Lin SL, Yeh CC, Wang JJ, Tao PL, et al. Amitriptyline suppresses neuroinflammationdependent interleukin-10-p38 mitogenactivated protein kinase-heme oxygenase-1 signaling pathway in chronic morphine-infused rats. Anesthesiology. 2009, 110:1379-1389. 
30. Sacerdote P. Effects of in vitro and in vivo opioids on the production of IL-12 and IL-10 by murine macrophages. Ann N Y Acad Sci 2003; 992:129-140.

31. Popiolek-Barczyk K, Kolosowska N, Piotrowska A, Makuch W, Rojewska E, Jurga AM, Pilat D, Mika J. Parthenolide Relieves Pain and Promotes M2 Microglia/Macrophage Polarization in Rat Model of Neuropathy. Neural Plast. 2015;2015:676473.

32. Qiu Z, Lu P, Wang K, Zhao X, Li Q, Wen J, Zhang H, Li R, Wei H, Lv Y, Zhang S, Zhang P. Dexmedetomidine Inhibits Neuroinflammation by Altering Microglial M1/M2 Polarization Through MAPK/ERK Pathway. Neurochem Res. 2020 Feb;45(2):345-353.

33. Zhang J, Wang Z, Wang Y, Zhou G, Li H. The effect of dexmedetomidine on inflammatory response of septic rats. 2015, BMC Anesthesiol 15:68.

34. Lin JP, Chen CQ, Huang LE, Li NN, Yang Y, Zhu SM, Yao YX. Dexmedetomldine attenuates neuropathic pain by inhibiting P2X7R expression and ERK phosphorylation in rats. 2018, Exp Neurobiol 27(4):267-276.

\section{Figures}




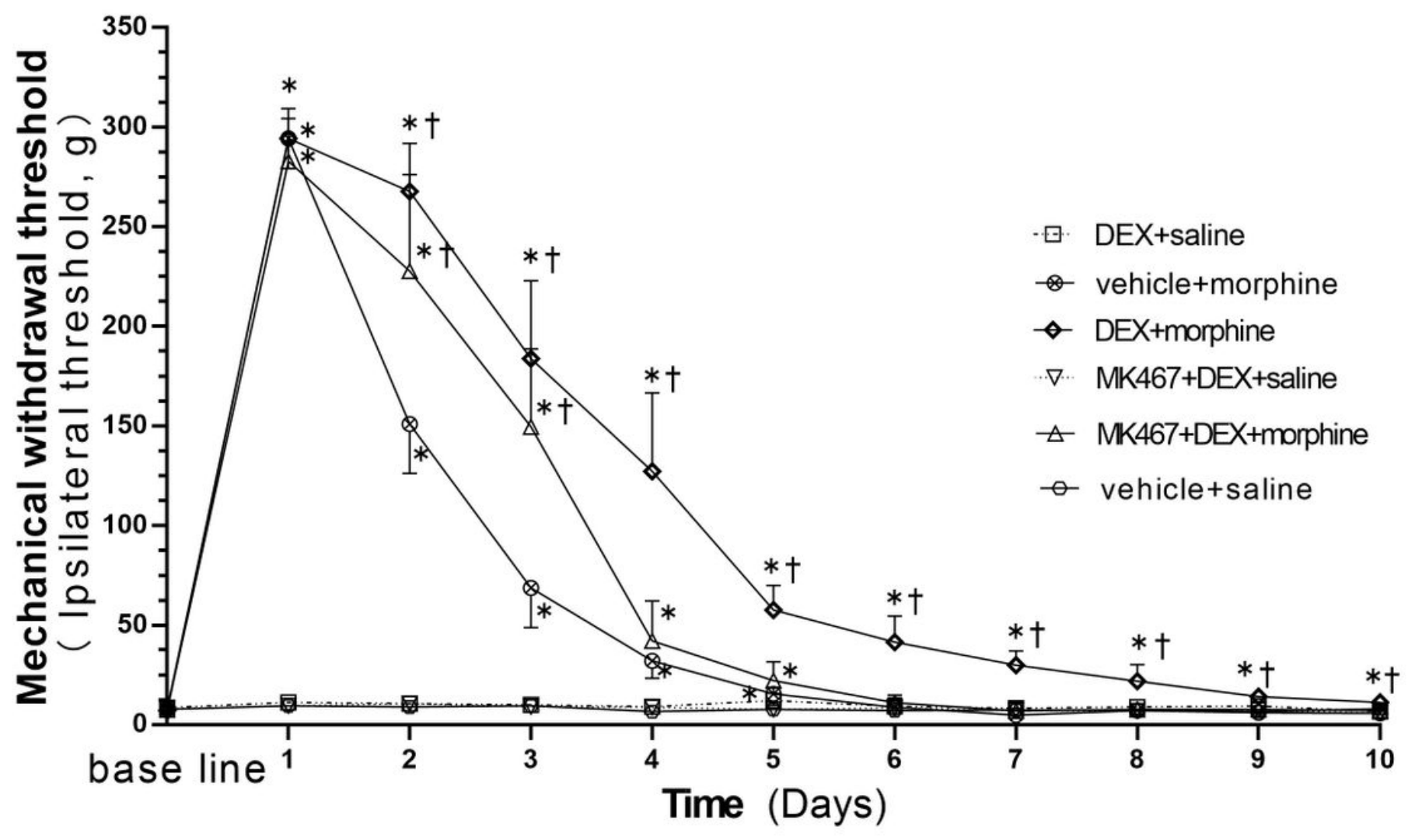

Figure 1

Mechanical withdrawal threshold (MWT) of ipsilateral hind paw in cancer pain rats.

Pre-injection (i.t.) of DEX attenuates mechanical hyperalgesia to repeated morphine administration. The rats $(n=6)$ received vehicle+saline, $D E X+$ saline, MK-467+DEX+saline, vehicle+morphine, $D E X+$ morphine, or MK-467+DEX+morphine respectively in each group, s.c. morphine or i.t. DEX or MK-467 injections twice daily for 10 days. The antinociceptive effect of the administered drugs was measured 30 minutes after the first injection (morning) via the von Frey test on each day. The values are presented as the mean $\pm S D$. A two-way repeated measures ANOVA in the MWT showed significant differences for the groups $(P<0.007)$, times $(P<0.007)$ and interaction $(P<0.007)$. Comparisons by Student $t$ test with Bonferroni correction ( $P<0.05 / 7=0.00714$ was defined as statistical significance) displayed significant within-group and between-group differences in MWT. ※P $<0.007$ compared with vehicle+saline group, $\# \mathrm{P}<0.007$ compared with vehicle+morphine group, ${ }^{*} \mathrm{P}<0.007$ compared with day 1 in corresponding group. S.C., subcutaneous; i.t., intrathecal; DEX, dexmedetomidine; MWT, mechanical withdrawal threshold. 


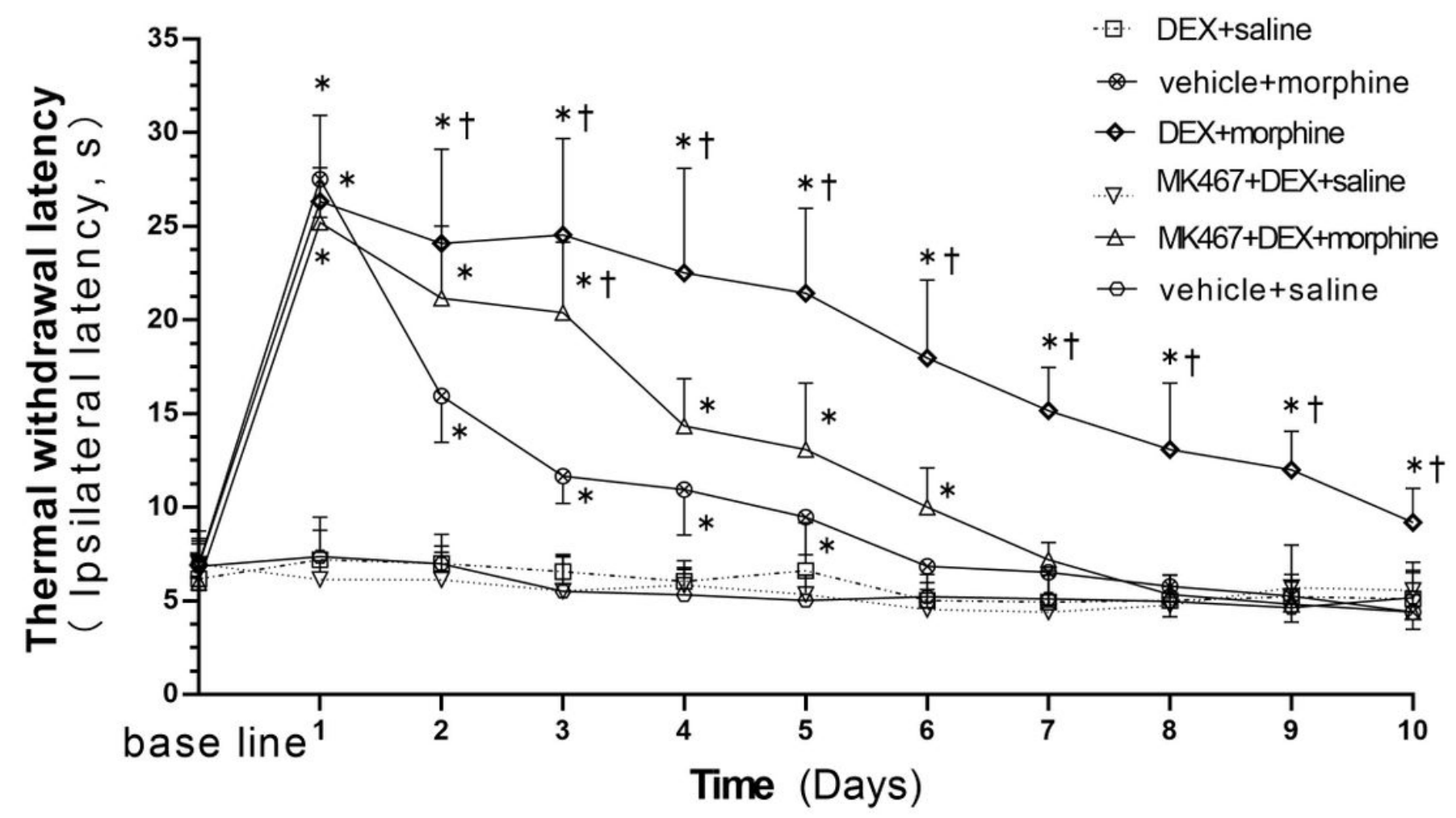

Figure 2

Thermal withdrawal latency (TWL) of ipsilateral hind paw in cancer pain rats.

Pre-injection (i.t.) of DEX attenuates thermal nociception to repeated morphine administration. The rats $(n=6)$ received vehicle+saline, DEX+saline, MK-467+DEX+saline, vehicle+morphine, DEX+morphine, or MK-

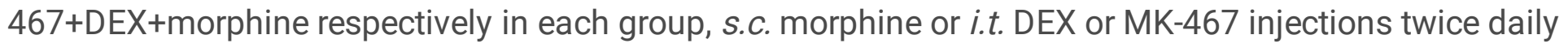
for 10 days. The antinociceptive effect of the administered drugs was measured 30 minutes after the first injection (morning) via the hot plate test on each day. The values are presented as the mean $\pm S D$. A twoway repeated measures ANOVA in the MWT showed significant differences for the groups $(P<0.007)$, times $(P<0.007)$ and interaction $(P<0.007)$. Comparisons by Student $t$ test with Bonferroni correction $(P<0.05 / 7=0.00714$ was defined as statistical significance) displayed significant within-group and between-group differences in TWL. $¥ \mathrm{P}<0.007$ compared with vehicle+saline group, $\# \mathrm{P}<0.007$ compared with vehicle+morphine group, ${ }^{*} \mathrm{P}<0.007$ compared with day 1 in corresponding group. S.c., subcutaneous; i.t., intrathecal; DEX, dexmedetomidine; TWL, thermal withdrawal latency. 
lba-1

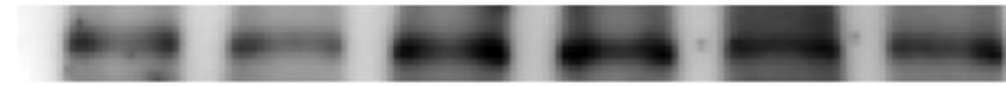

GAPDH
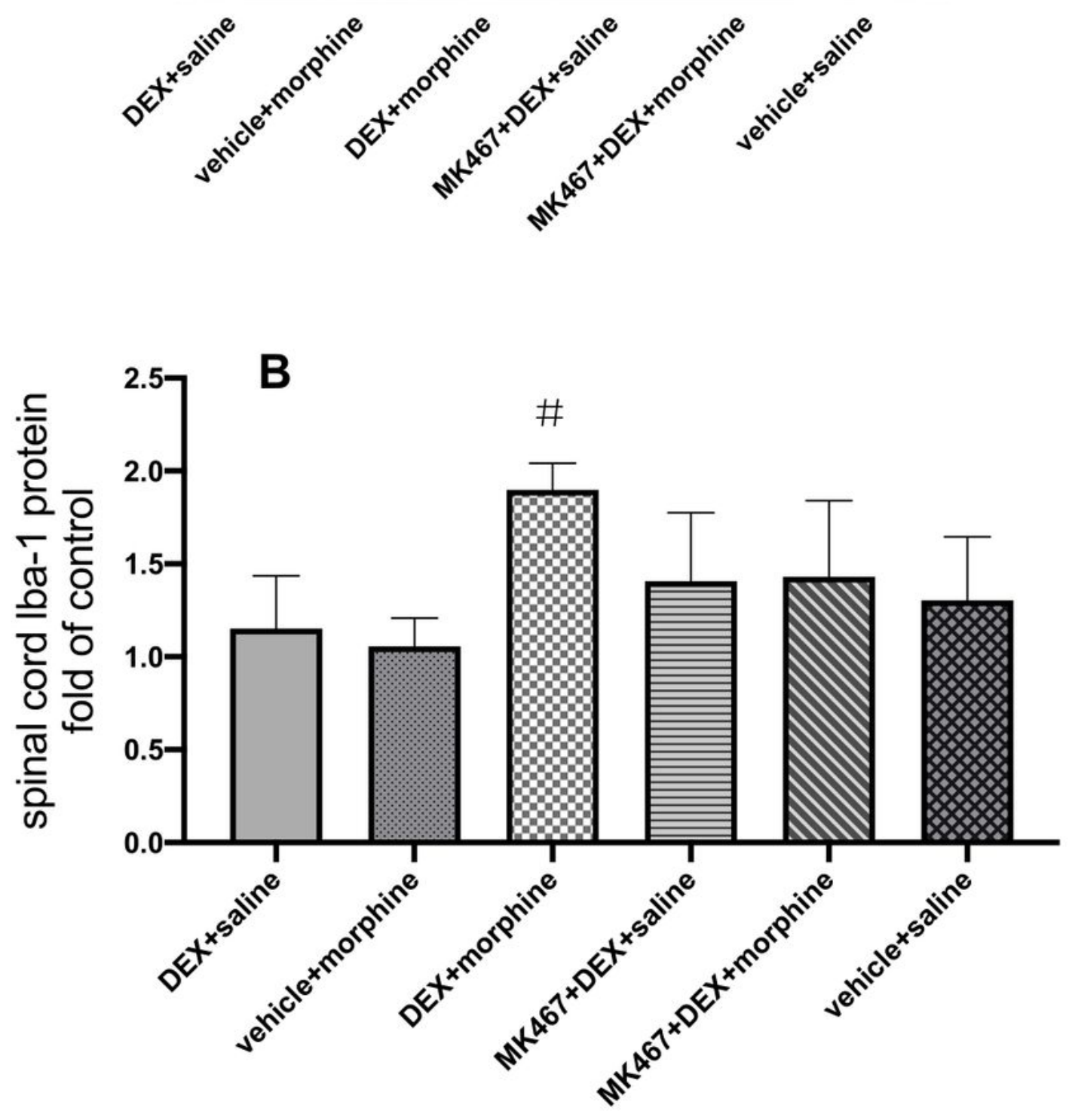

Figure 3

Effects of i.t. injection of DEX on Ibal-1 protein expression in ipsilateral spinal cord of morphine tolerant rats with cancer pain. (A) protein expression, (B) analysis of gray value of protein bands. Western blotting was performed using the ipsilateral lumbar L3/L4/L5 segments of DRG. The data are expressed as the mean fold-change protein expression relative to the mean $\pm S D(n=3)$. Vehicle+saline group served as control. The protein expression levels were analyzed via a one-way analysis of variance ANOVA and then 
followed by multiple comparisons using Student $t$ test with Bonferroni correction ( $P<0.05 / 7=0.007143$ was defined as statistical significance). $\# \mathrm{P}<0.007$ compared with vehicle+morphine group. i.t., intrathecal; DEX, dexmedetomidine; DRG, dorsal root ganglia.
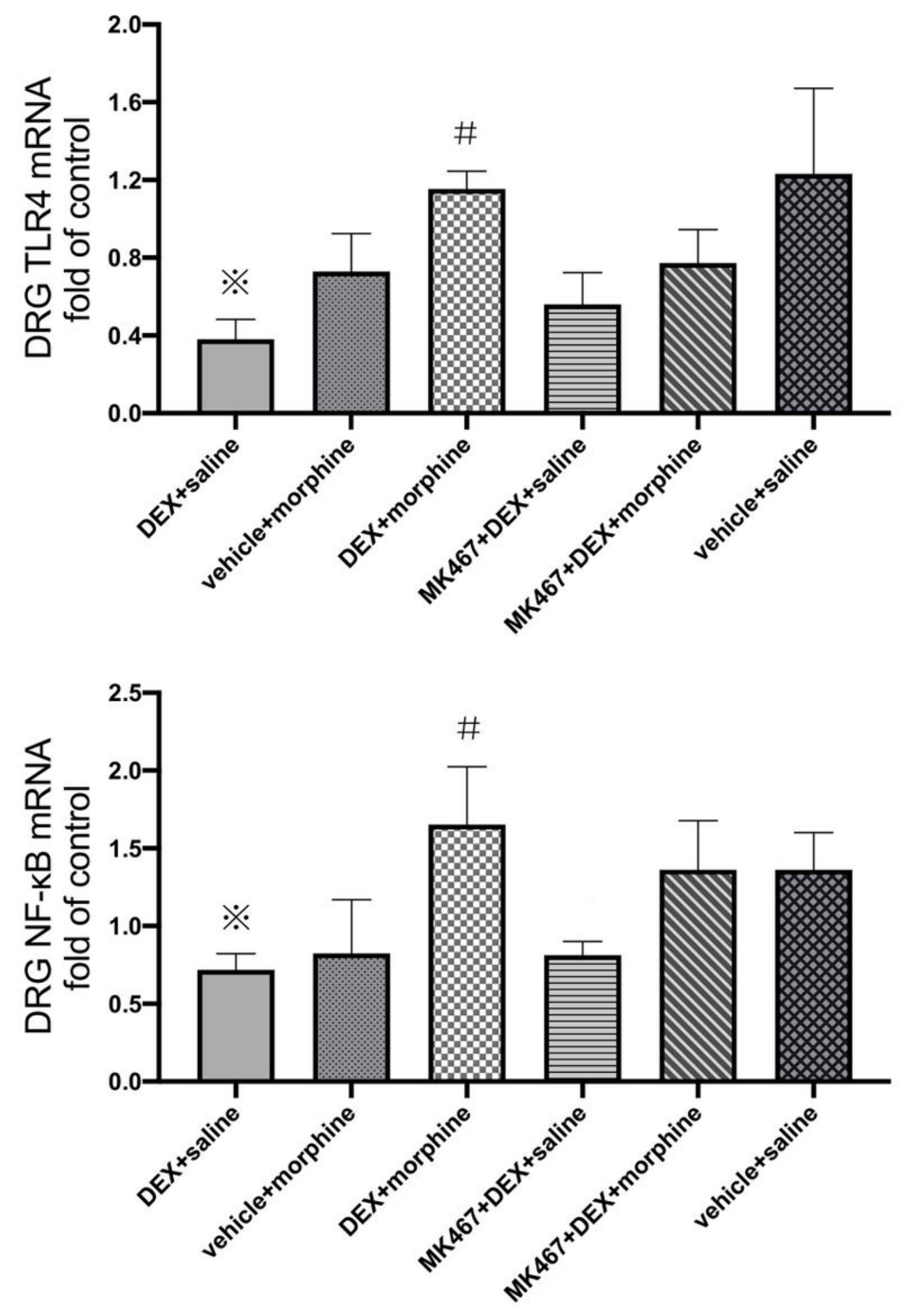

Figure 4 
Effects of i.t. injection of DEX on TLR4 (Fig. 3-1) and NF-KB (Fig. 3-2) mRNA expression in ipsilateral DRG of morphine tolerant rats with cancer pain. The data are expressed as the mean fold-change in $\mathrm{mRNA}$ expression relative to the mean $\pm S D(n=6)$. Vehicle+saline group served as control. The mRNA expression levels were analyzed via a one-way analysis of variance ANOVA $(P=0.001)$ and then followed by multiple comparisons using Student $t$ test with Bonferroni correction $(P<0.05 / 7=0.007143$ was defined as statistical significance). $※ \mathrm{P}<0.0071$ compared with vehicle+saline group, $\# \mathrm{P}<0.007$ compared with vehicle+morphine group. i.t., intrathecal; DEX, dexmedetomidine; DRG, dorsal root ganglia.
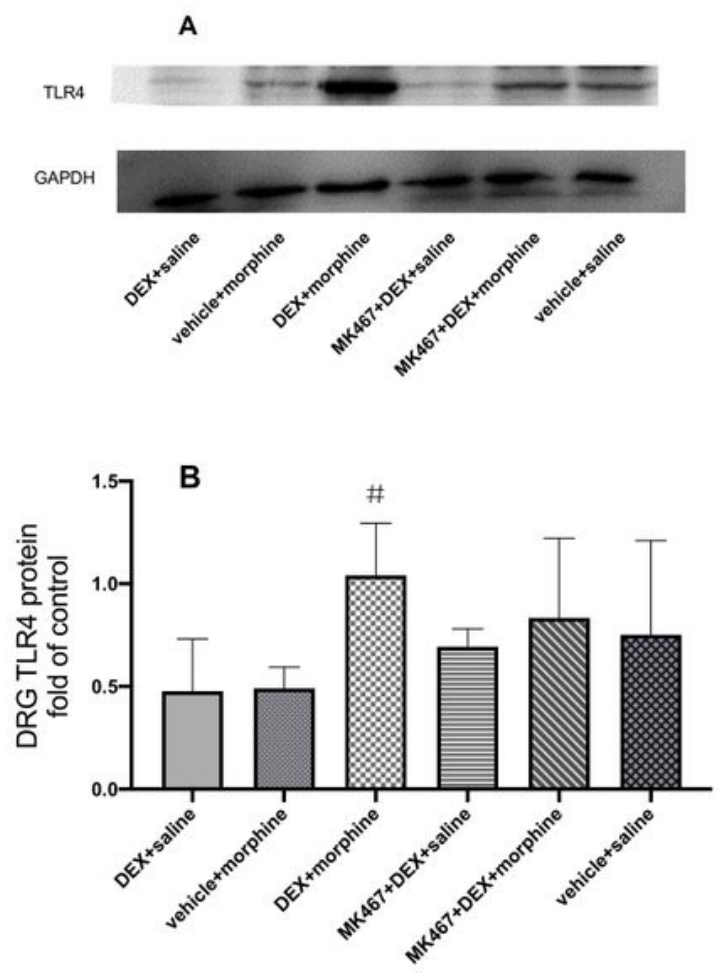

A
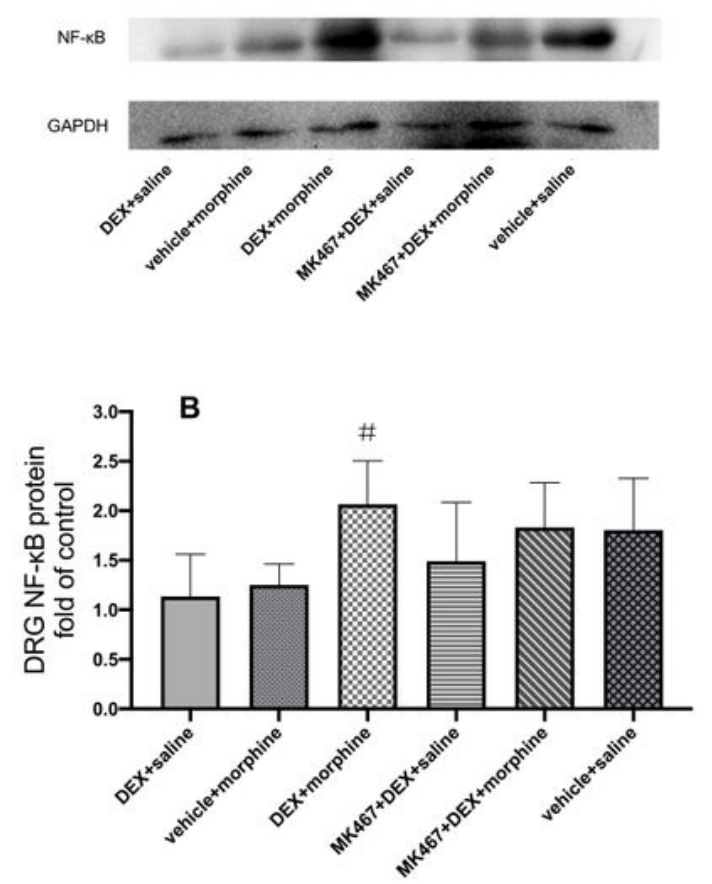


\section{Figure 5}

Effects of i.t. injection of DEX on TLR4 (Fig. 4-1) and NF-KB (Fig. 4-2) protein expression in ipsilateral DRG of morphine tolerant rats with cancer pain. (A) protein expression, (B) analysis of gray value of protein bands. Western blotting was performed using the ipsilateral lumbar L3/L4/L5 segments of DRG. The data are expressed as the mean fold-change protein expression relative to the mean $\pm S D(n=6)$. Vehicle+saline group served as control. The protein expression levels were analyzed via a one-way analysis of variance ANOVA and then followed by multiple comparisons using Student $t$ test with Bonferroni correction ( $P<0.05 / 7=0.007143$ was defined as statistical significance). $\# P<0.007$ compared with vehicle+morphine group. i.t., intrathecal; DEX, dexmedetomidine; DRG, dorsal root ganglia. 

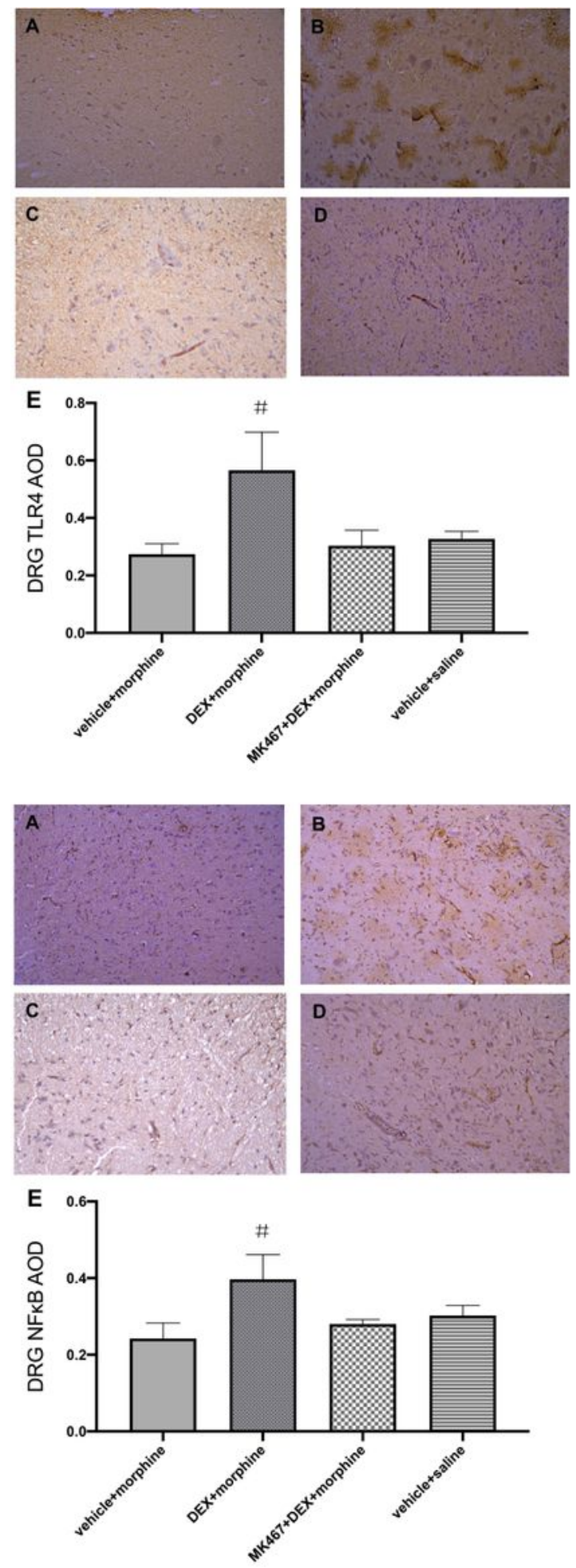

\section{Figure 6}

Immunohistochemical staining of TLR4 (Fig. 5-1) and NF-KB (Fig. 5-2) protein in DRG sections. (A) vehicle+morphine group. (B) DEX+morphine group. (C) DEX+MK-467+morphine group. (D) vehicle+saline group. (E) AOD in slices of DRG. The values are presented as the mean $\pm S D(n=4)$. Scale bar, $50 \mu \mathrm{m}$. The protein expression levels were analyzed via a one-way analysis of variance ANOVA and then followed by multiple comparisons using Student $t$ test with Bonferroni correction $(P<0.05 / 5=0.01$ was defined as 
statistical significance). \#P<0.01 compared with vehicle+morphine group. i.t., intrathecal; DEX, dexmedetomidine; DRG, dorsal root ganglia. AOD, average optical density.
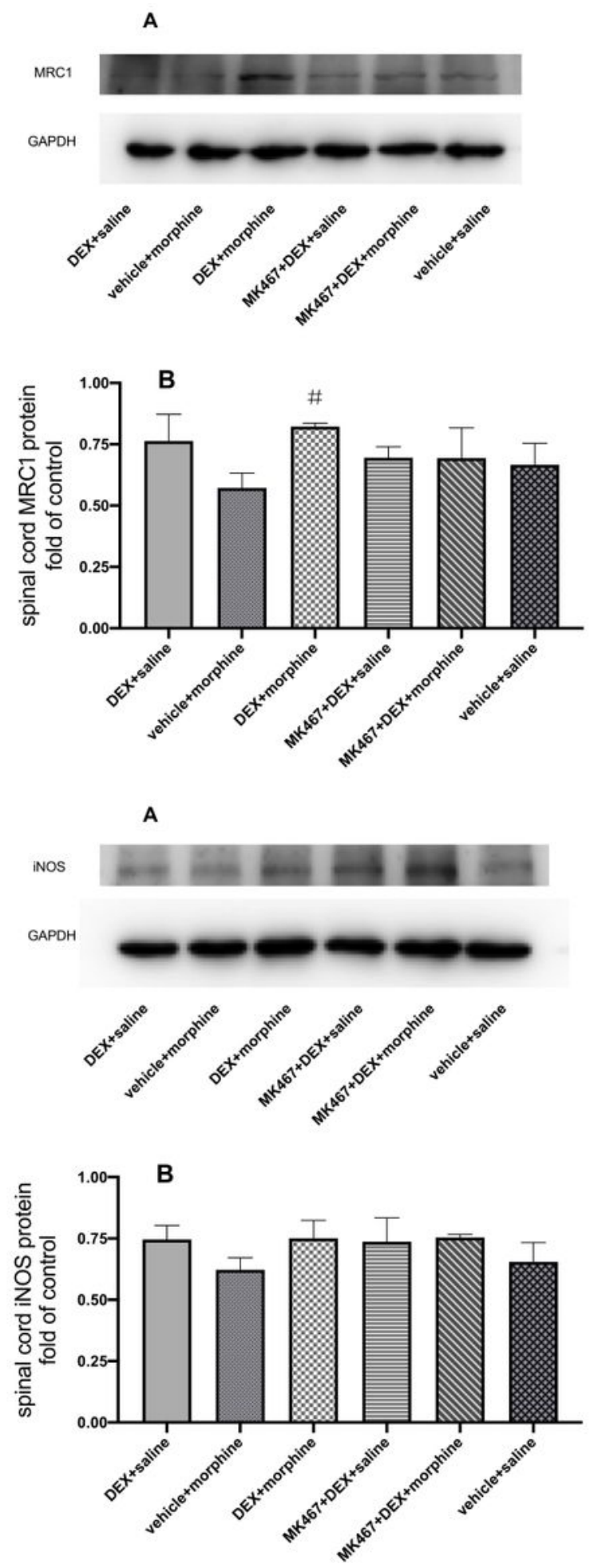

Figure 7

Effects of i.t. injection of DEX on CD206 (Fig. 6-1) and iNOS (Fig. 6-2) protein expression in ipsilateral SC of morphine tolerant rats with cancer pain. (A) protein expression, (B) analysis of gray value of protein 
bands. Western blotting was performed using the ipsilateral lumbar L3/L4/L5 segments of spinal cord. The data are expressed as the mean fold-change protein expression relative to the mean $\pm S D(n=3)$. Vehicle+saline group served as control. The protein expression levels were analyzed via a one-way analysis of variance ANOVA and then followed by multiple comparisons using Student $t$ test with Bonferroni correction ( $P<0.05 / 7=0.007143$ was defined as statistical significance). $\# P<0.007$ compared with vehicle+morphine group. i.t., intrathecal; DEX, dexmedetomidine; SC, spinal cord.
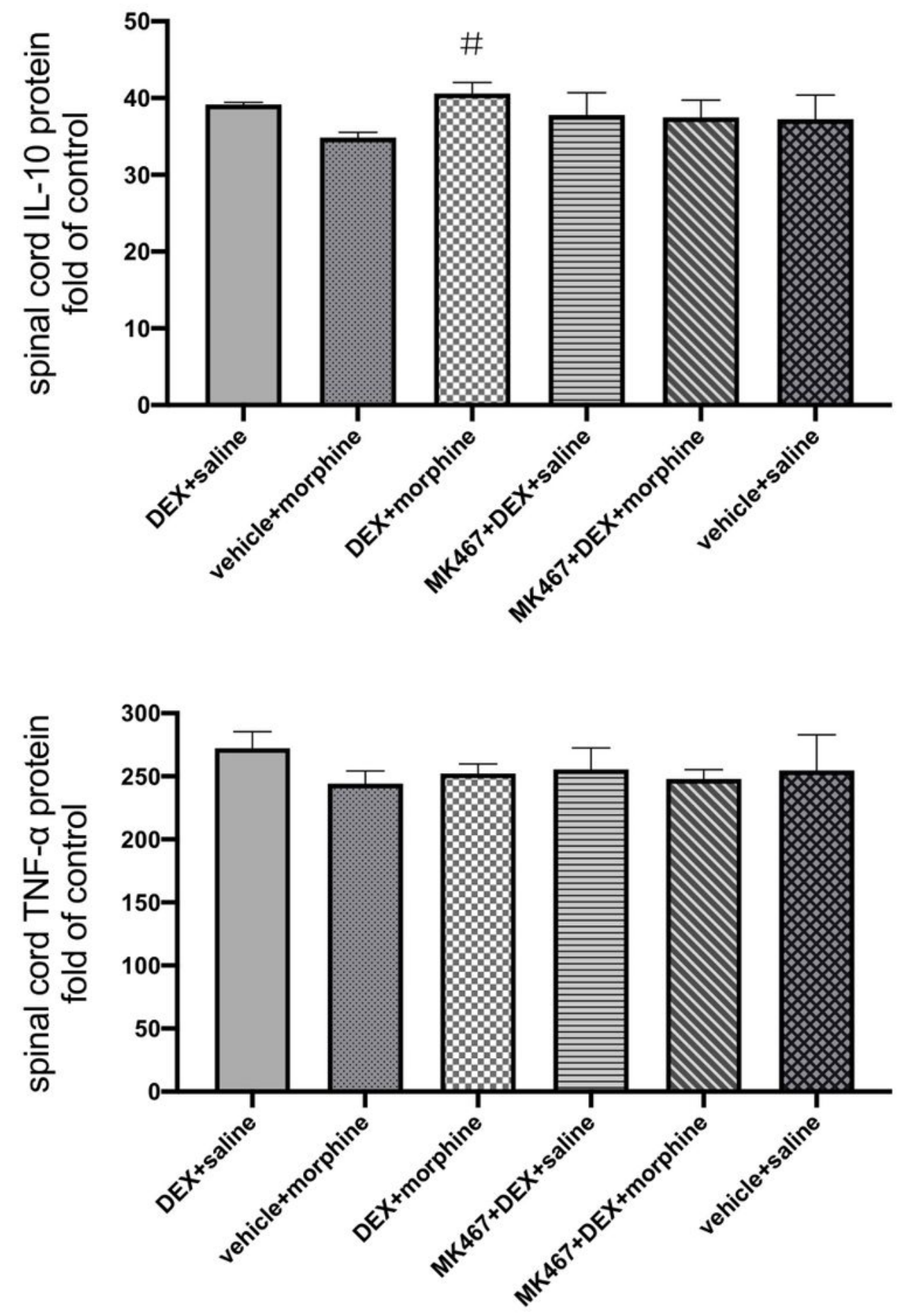


\section{Figure 8}

Effects of i.t. injection of DEX on IL-10 (Fig. 7-1) and TNF-a (Fig. 7-2) protein expression in ipsilateral SC of morphine tolerant rats with cancer pain. ELISA was performed using the ipsilateral lumbar L3/L4/L5 segments of spinal cord. The data are expressed as the mean fold-change protein expression relative to the mean $\pm S D(n=3)$. Vehicle+saline group served as control. The protein expression levels were analyzed via a one-way analysis of variance ANOVA and then followed by multiple comparisons using Student $t$ test with Bonferroni correction ( $P<0.05 / 7=0.007143$ was defined as statistical significance). \#P<0.007 compared with vehicle+morphine group. i.t., intrathecal; DEX, dexmedetomidine; SC, spinal cord. 